NBER WORKING PAPER SERIES

SYMMETRIC SUBSTITUTION MATRICES

IN ASSET DEMAND SYSTEMS

David S. Jones

Working Paper No. 574

NATIONAL BUREAU OF ECONOMIC RESEARCH

1050 Massachusetts Avenue

Cambridge MA 02138

October 1980

This paper is part of the NBER's research program in Financial Markets and Monetary Economics. Any opinions, findings or conclusions expressed herein are those of the author and do not necessarily reflect the views of the National Bureau of Economic Research. 


\title{
Symmetric Substitution Matrices in Asset Demand Systems
}

\begin{abstract}
In this paper, necessary and sufficient conditions for an asset substitution matrix to be symmetric for all distributions of rates of return are derived. It is found that symmetry in this context is essentially equivalent to the proposition that the von Neumann-Morgenstern utility function displays either constant absolute or constant relative risk aversion, depending upon whether the substitution matrix is defined in terms of arithmetic or geometric rates of return.
\end{abstract}

David S. Jones Department of Economics Northwestern University Evanston, Illino1s 60201

(312) $492-5690$ 


\section{Symmetric Substitution Matrices in Asset Demand Systems*}

\section{Introduction}

Consider the generic single-period portfolio choice problem in which an investor seeks to allocate wealth " $\mathrm{W}_{0}$ " among " $\mathrm{k}$ " alternative assets so as to maximize the expected utility of end-of-period wealth. Denoting the amount of money invested in the $i^{\text {th }}$ asset by $A_{i}$ in the absence of transactions costs this problem may be formally written as:

$$
\begin{aligned}
& \operatorname{maximize} E\left\{U\left[{\stackrel{\mathbf{E}}{\sum_{i}} A_{i}}_{R_{i}} R^{j}\right\}\right. \\
& A_{1} \ldots, A_{k} \\
& \text { subject to: } \sum_{i=1}^{k} \mathbb{A}_{i}=W_{0}
\end{aligned}
$$

where $E\{\cdot\}=$ the expectation operator,

$$
\begin{aligned}
\mathrm{U}[\cdot]= & \text { a von Neumann - Morgenstern utility function } \\
& \text { displaying non-satiation and risk aversion } \\
& \left(\text { i.e., } \mathrm{U}^{\prime}>0 \text { and } \mathrm{U}^{\prime \prime}<0\right), \text { and } \\
\tilde{R}_{\mathrm{i}}= & \text { the random gross after tax rate of return per }
\end{aligned}
$$

It will prove convenient below to parameterize the random rates of return $\widetilde{\mathrm{R}}_{i}$ in the form:

$$
\begin{aligned}
\mathrm{R}_{i} & \equiv 1+\tilde{\mathbf{r}}_{i} \\
& \equiv 1+\overline{\mathbf{r}}_{i}+\tilde{\mathrm{x}}_{i}
\end{aligned}
$$

where $\quad \tilde{r}_{i}=$ the net rate of return per dollar invested in the $i^{\text {th }}$ asset,

$\bar{r}_{i}=E\left\{\tilde{r}_{i}\right\}$, and

$\tilde{\mathbf{x}}_{j}=$ a zero mean randam variable definitionally set equal to the prediction error in $\tilde{r}_{i}$.

Substituting expression (2) into (1), we can in principle solve the portfolio choice problem for the optimal asset demands $A_{i}^{*}$. These 
asset demands are generally of the form:

$$
A_{i}^{\star}=a_{i}\left[\tilde{r}_{i}, \ldots, \tilde{r}_{k} ; \Phi ; w_{0}\right]
$$

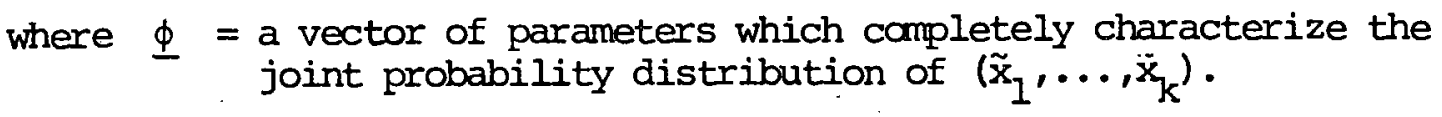

The asset substitution matrix "S" is, by definition, the Jacobian of the system of asset demand equations (3) with respect to the vector of net rates of return. Thus, the typical element of $S$ is:

$$
s \equiv\left[s_{i j}\right]=\left[\frac{\partial \stackrel{\star}{A}_{i}}{\partial \bar{r}_{j}}\right] \text {. }
$$

Without prior information about either the form of the utility function $\mathrm{U}[\cdot]$ and/or the joint probability distribution of rates of return preciously little can be said about the structure of $S$ other than that each of its columns sum to zero and that its diagonals are likely to be positive. ${ }^{1}$ This lack of knowledge about the structure of $S$ is sometimes not well appreciated. For example, it is traditional in the monetary economics literature to say that asset " $i$ " is a substitute (complement) to asset " $j "$ if the sign of $s_{i j}$ is negative (positive). In general, however, these concepts are not well defined because the sign of $s_{i j}$ need not be the same as the sign of $s_{j i}$ for $i \neq j .^{3}$ Thus, we might have the confusing result that asset " $i$ " is a substitute for asset " $j$ " but that " $j$ " is a complement to " $i$ ".

One situation in which the above ambiguity does not arise is when the substitution matrix is symmetric. Under symmetry $s_{i j}=s_{j i}$ implying that the cross yield effects between two assets are equal and therefore of the same sign.

Symmetry of the substitution matrix not only pemits one to define asset substitutability and complementarity unambiguously, it has useful empirical implications as well. Gramlich and Kalchbrenner (1970) were the earliest to observe the fact that symetry of the substitution matrix 
may facilitate the estimation of systems of asset demand equations which are linear in expected rates of return. To appreciate this fact, consider the following simplified vector system of asset demand equations

$$
\underline{A}^{*}=(B \underline{\bar{r}}+\underline{d}) \cdot W_{0}
$$

where $\mathrm{B}$ is a $\mathrm{k} \times \mathrm{k}$ matrix of coefficients and $\underline{\mathrm{d}}$ is a $\mathrm{k} \times \mathrm{l}$ vector of coefficients. In general, there are $k^{2}-1$ independent parameters to be estimated after allowances are made for the balance sheet constraint. If the substitution matrix $B \cdot W_{0}$ is symetric, however, the number of independent parameters is reduced to $\left(k^{2}+k-2\right) / 2$. For $k=5$, this results in a decline in the number of coefficients to be estimated from 24 to 14 :

Symmetry restrictions on the substitutinn matrix have heen usofully employed in empirical estimations of systems of asset demands by Gramlich and Kalchbrenner (1970), and Hendershott (1977). Smith (1978), has criticized this practice for apparently lacking theoretical justification ${ }^{4}$. The purpose of the present paper is to provide a theoretical justification for imposing symmetry restrictions on the substitution matrix within the context of the portfolio choice model discussed above. Specifically, in Section II of this paper we shall derive necessary and sufficient conditions for the substitution matrix to be symmetric for all joint probability distributions of rates of return. Concluding remarks are presented in Section III. 
$-4-$

II Symmetry of the Substitution Matrix

II.A Case 1: Discrete Compounding

Roley (1977) was the first to derive necessary and sufficient conditions for the substitution matrix to be symmetric in a portfolio choice setting with uncertainty. His analysis, though, is confined to mean-variance models of investor behavior. Nonetheless, his results are useful as a starting point in our investigation of the symmetry property for general von Neumann - Morgenstern expected utility maximizers.

Suppose that an investor has a mean-variance utility function of the form $u\left[\mu, \sigma^{2}\right]$ where $\mu$ and $\sigma^{2}$ are the mean and variance of end-of-period wealth respectively. A measure of the investor's risk aversion is $-u_{1} / u_{2}$, defined to be the investor's mean-variance absolute risk aversion. Roley proves that a necessary and sufficient condition for a mean-variance investor's substitution matrix to be exactly symmetric is that the investor display constant mean-variance absolute risk aversion.

The first new rèsult of this páper is to generalize Roley's theorem to von Neumann-Morgenstern expected utility maximizers:

Theorem 1: Consider the portfolio choice problem (1)whose solution is given in (3). Then a necessary and sufficient condition for the substitution matrix $S$ to be symetric for all joint probability distributions of rates of return is that utility display constant absolute risk aversion. 5

Clearly Theorem 1 includes Roley's result as a special case when meanvariance preferences are derived from an underlying von Neumann Morgenstern utility function. 
Same care must be exercised when interpretting Theoreml. When utility displays constant absolute risk aversion this theorem says that the substitution matrix will be symetric regardless of the joint probability distribution of rates of return. It also says that only this type of utility function generates a symmetric substitution matrix for all joint distributions of rates of return. Theorem 1 does not claim, however, that the substitution matrix will never be symmetric if utiltiy does not display constant absolute risk aversion: given an arbitrary utility function it may be possible to find some distribution of rates of return that will generate a symetric substitution matrix.

\section{II.B Case 2: Continuous Compounding}

Constant absolute risk aversion is a very stringent assumption about investor behavior which does not have much empirical support. Therefore, empirical applicability of Theorem 1 is likely to be quite limited.

Constant relative risk aversion, on the other hand, appears to characterize the preferences of many groups of investors reasonably well. 6 In the present section we shall redefine the substitution matrix in terms of geametric rather than arithmetic rates of return. Having done this it is straightforward to derive a result analogous to Theorem 1 in which constant relative risk aversion is both necessary and sufficient for the (redefined) substitution matrix to be symetric.

Rather than parameterize gross rates of return $\tilde{R}_{i}$ by the arithmetic net rates of return as in (2), it is some.times more convenient to parameterize them by their geametric analogues $\tilde{g}_{i}$ defined by
(2')
$\tilde{R}_{i} \equiv \exp \left[\tilde{g}_{i}\right]$ 


$$
\equiv: \exp \left[\bar{g}_{i}+\tilde{y}_{i}\right]
$$

where $\tilde{g}_{i}=$ the net geametric rate of return per dollar invested in the $i^{\text {th }}$ asset,

$$
\begin{aligned}
& \bar{g}_{i}=E\left\{\tilde{g}_{i}\right\}, \text { and } \\
& \tilde{y}_{i}=\tilde{g}_{i}-\bar{g}_{i} .
\end{aligned}
$$

Essentially, $\tilde{g}_{i}$ differs from $\tilde{r}_{i}$ in that the former is the net rate of return per unit time if rates of return are compounded continuously during the investment period whereas the former is the appropriate concept if returns are compounded only at the end of the investment period.

Analogous to (3), the solution to the portfolio choice problem generated by substituting (2') into (1) can be written in the general form:

$$
\stackrel{\star}{\mathrm{A}}_{i}=a_{i}\left[\bar{g}_{1}, \ldots, \bar{g}_{k} ; \underline{\Psi} ; w_{0}\right]
$$

where

$$
\begin{aligned}
\Psi= & \text { a vector of parameters which completely } \\
& \text { characterize the joint probability } \\
& \text { distribution of }\left(\tilde{\mathrm{Y}}_{1}, \ldots, \tilde{\mathrm{Y}}_{\mathrm{k}}\right) .
\end{aligned}
$$

We are interested in the conditions under which the (geometric) substitution matrix defined by

$$
\Omega \equiv\left[\Omega_{i j}\right] \equiv\left[\frac{\partial \mathrm{A}_{i}{ }^{*}}{\partial \tilde{g}_{j}}\right]
$$

is symmetric. Our results are summarized in the following theoren which is proved in Appendix 2:

Theorem 2: A necessary and sufficient condition for $\Omega$ to be symmetric for all joint probability distributions of rates of return is that utility display constant relative risk aversion.

This theorem should be interpreted in the same manner as was described for Theorem 1. 7 
III Concluding Remarks

Above we have derived necessary and sufficient conditions for the substitution matrix to be symetric, depending on whether this matrix is defined in terms of aritmetic or geometric net rates of return. In the former case constant absolute risk aversion is required for symmetry whereas in the latter case it is constant relative risk aversion.

Several limitations of our analysis are apparent. First, the portfolio choice framework of this study, given in (1), abstracts from transactions costs. As such, the substitution matrices discussed above are best interpreted as long-run or equilibrium substitution matrices.

A question naturally arises about the symmetry of the short-run substitution matrix when transactions costs are incorporated into the analysis. It is proven in Appendlx 3 that when transactions costs are incorporated into the above portfolio choice framework then the (arithmetic) short-run substitution matrix is symmetric if utility displays constant absolute risk aversion regardless of the form of the transactions cost function, provided that it is twice differentiable.

The above portfolio choice paradıgm is also ınherently static. The introduction of dynamic considerations into this framework, however, renders the problem exceeding $\perp y$ difficult and well beyond the intended scope of tnis paper. 


\section{Footnotes}

*This paper is a portion of my thesis which was written under the supervision of Professors Benjamin Friedman and John Lintner. Their comments have been greatly appreciated.

1. See Jones (1979) for a discussion of when the diagonals are positive. In general, $S_{i i}>0$ if $\frac{\partial A_{j}^{*}}{\partial W_{0}}>0$ and $A_{i}^{*}>0$.

2. See for instance, Silber (1970).

3. This case may result if one of $\frac{\partial A_{j}}{\partial W_{0}} \cdot A_{i}{ }^{*}$ and $\frac{\partial A_{i}{ }^{*}}{\partial \bar{w}_{0}} \cdot A_{j}^{*}$ is quite small and the other is quite large and positive. See Jones (1979) for a discussion.

4. Roley (1977) has provided a rigorous justification for the symmetry restrictions for mean-variance investors. See section II below.

5. See Appendix 1 for a proof of Theorem 1. See Jones (1979) for a proof which emphasizes the similarities between portfolio theory and neoclassical consumption theory.

6. See Jones (1979) and the references cited therein.

7. When a riskless asset exists then the following interesting result obtains: that portion of the arithmetic (geometric) substitution matrices corresponding to just the risky assets is symmetric for all distributions of rates of return if and only if utility displays hyperbolic absolute risk aversion in the sense of Merton (1972). See Jones (1979) for a proof of this result. 
APPENDIX 1

Proof of Theorem 1:

Necessity:

Step 1: After using the wealth constraint to rewrite

problem $(1)$ in terms of the unconstrained choice over $A_{1}, \ldots, A_{k-1}$ the first order conditions are

(Al) $\quad E\left\{U^{\prime} \cdot\left(\tilde{R}_{i}-\tilde{R}_{k}\right)\right\}=0 \quad$ for $i=1, \ldots, k-1$.

Step 2: Since each column of $S$ sums to zero, by virtue of the wealth constraint, symmetry implies that if all $\bar{r}_{i}$ are increased by " $\delta$ " then all asset demands remain unchanged. BY totally differentiating (AI) and assuming that the Hessian associated with $(A I)$ is positive definite so that the second order conditions hold this is seen to imply that

$$
E\left\{U^{\prime \prime} \cdot\left(\tilde{R}_{i}-\tilde{R}_{k}\right)\right\}=0 \quad \text { for } i=1, \ldots, k-1
$$

Moreover, because symmetry must hold for all joint distributions of the $\tilde{R}_{i}$, (A2) implies that $U^{\prime \prime}$ and $U^{\prime}$ are proportional. This is the same as saying that U.[-] displays constant absolute risk aversion.

\section{Sufficiency:}

Step 1: Define the matrix $\mathrm{H}$ to be the Hessian associated with (AI). Thus, 
(A3) $\quad H \equiv\left[H_{i j}\right]=\left[E\left\{U^{\prime \prime} \cdot\left(\tilde{R}_{i}-\tilde{R}_{k}\right)\left(\tilde{R}_{j}-\tilde{R}_{k}\right)\right\}\right]$.

Also define

(A4)

$$
\hat{\mathrm{s}} \equiv\left(\begin{array}{lll}
\frac{\partial \mathrm{A}_{1}^{*}}{\partial \bar{r}_{1}} & \cdot & \frac{\partial \mathrm{A}_{1}^{*}}{\partial \bar{r}_{\mathrm{k}-1}} \\
\cdot & & \\
\cdot & & \\
\frac{\partial \mathrm{A}_{\mathrm{k}-1}^{*}}{\partial \bar{r}_{1}} & \cdot & \frac{\partial A_{k-1}^{*}}{\partial \bar{r}_{k-1}}
\end{array}\right) \cdot
$$

The matrix $\hat{\mathbf{S}}$ is therefore the upper $(k-1) \times(k-1)$ submatrix of S (which we will recall is $k \mathrm{x} k$ ).

Step 2: Totally differentiating (AI) and making use of the assumption of constant absolute risk aversion we obtain (A5) $\quad \hat{\mathrm{S}}=-\mathrm{E}\left\{\mathrm{U}^{\prime}\right\} \cdot \mathrm{H}^{-1}$.

Hence, $\hat{S}$ is symmetric. Moreover, since the choice of which asset to eliminate via the wealth constraint was arbitrary, it must be true that $\mathbf{S}$ too is symmetric. 


\section{APPENDIX 2}

Proof of Theorem 2:

\section{Necessity:}

Step 1: Since each column of the substitution matrix

$\Omega$ sums to zero, symmetry implies that each row must also sum to zero. That is, an equivalent increase in all $\bar{g}_{i}$ leaves all asset demands unchanged. Equivalently, all asset demands are unchanged if all gross yields $\tilde{R}_{i}$ are increased by the same proportional amount " $\beta . "$

This can be shown to imply that

(A6) $\quad E\left\{U^{\prime \prime} \cdot\left[\sum_{i=1}^{k} A_{i}\left(\tilde{R}_{i}-\tilde{R}_{k}+w_{0} \tilde{R}_{k}\right)\left(\tilde{R}_{i}-\tilde{R}_{k}\right)\right]\right\}=0$ for $i=1, \ldots, k-1$. Since this must hold for all joint distributions of the $\tilde{R}_{i}$ it must be true that $U^{\prime}$ and $U^{\prime \prime} \cdot\left(\sum_{i=1}^{k} A_{i}\left(\tilde{R}_{i}-\tilde{R}_{k}\right)+w_{0} \tilde{R}_{k}\right)$ are proportional. This, however, is equivalent to the requirement that $\mathrm{U}[\cdot]$ displays constant relative risk aversion.

\section{Sufficiency:}

Step 1: Define

(A7)

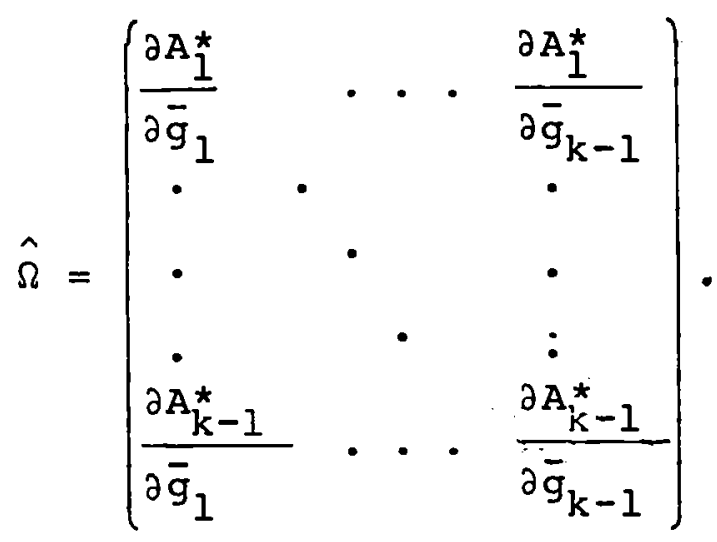


Totally differentiating (Al) and invoking constant relative risk aversion yields

(A8) $\hat{\Omega}=-E\left\{U^{\prime} \cdot \tilde{R}_{k}\right\} H^{-1}-\left(\begin{array}{ccc}A_{1}^{*} & \cdot & 0 \\ \cdot & & \\ & \cdot & \\ 0 & \cdot & A_{k-1}^{*}\end{array}\right)+\frac{1}{W_{0}} \cdot\left(\begin{array}{l}A_{1}^{*} \\ \cdot \\ \cdot \\ A_{k-1}^{*}\end{array}\right) \cdot\left(\begin{array}{l}A_{1}^{*} \\ \cdot \\ \cdot \\ A_{k-1}^{*}\end{array}\right)^{T}$

which is clearly symmetric. Since the choice of which asset to drop via the wealth constraint was arbitrary, this implies that $\Omega$ is also symmetric. 


\section{APPENDIX 3}

Within the framework described above transactions costsmay be incorporated by positing that costs $c\left[A_{1}, \ldots, A_{k}\right]$ are incurred if the beginning-of-period portfolio is $\left(A_{1}, \ldots, A_{k}\right)$. Conceptually, the scalar cost function $c[\cdot]$ is also parameterized by the asset holdings inherited or carried forward from the previous period. Since these are given at the time the current portfolio decision is made, these additional parameters will be excluded from our notation for simplicity.

Thus, the portfolio choice problem with transactions costs may be written

(A9) $\underset{A_{1}, \ldots, A_{k}}{\text { Maximize }} E\left\{U\left[\sum_{i=1}^{k} A_{i} \tilde{R}_{i}\right]\right\}$

subject to

$$
\sum_{i=1}^{k} A_{i}+c\left[A_{1}, \ldots, A_{k}\right]=w_{0} .
$$

Under general conditions the portfolio holding of the $\mathrm{k}^{\text {th }}$ asset can be solved as a function of $A_{1}, \ldots, A_{k-1}$ and $W_{0}$ from the wealth constraint in (A9). Denote this function

$$
A_{k}=f\left[A_{1}, \ldots, A_{k-1} ; W_{0}\right]
$$

Then the portfolio choice problem may be rewritten as an unconstrained optimization over $A_{1}, \ldots, A_{k-1}$ : 
(A11) $\underset{A_{1}, \ldots, A_{k-1}}{\operatorname{Maximize}} E\left\{U\left[\sum_{i=1}^{k} A_{i}\left(\tilde{R}_{i}-\tilde{R}_{k}\right)+f\left[A_{1}, \ldots, A_{k-1} ; W_{0}\right] \tilde{R}_{k}\right]\right\}$ The first order conditions are:

(A12) $\quad E\left\{U^{\prime} \cdot\left[\left(\tilde{R}_{i}-\tilde{R}_{k}\right)+\frac{\partial f}{\partial A_{i}} \tilde{R}_{k}\right]\right\}=0 \quad$ for $i=1, \ldots, k-1$.

Define the short-run substitution matrix to be

(A13) $\quad\left[\equiv\left[L_{i j}\right] \equiv\left[\frac{\partial A_{i}^{*}}{\partial \bar{r}_{j}}\right]\right.$

where $A_{k}^{*} \equiv f\left[A_{1}^{*}, \ldots, A_{k-1}^{*} ; W_{0}\right]$. Also, let $\hat{\sum}$ be the upper lefthand $(k-1) \times(k-1)$ submatrix of $\sum$. Then totally differentiating (A12) yields :

(A14) $\hat{L}=-M^{-1}\left[E\left\{U^{\prime}\right\} I+E\left\{U^{\prime \prime} \cdot\left[\begin{array}{l}\tilde{R}_{1} \\ \cdot \\ \tilde{R}_{k-1}\end{array}\right)-\left(\begin{array}{l}\tilde{R}_{k} \\ \dot{R_{k}} \\ \tilde{R}_{k}\end{array}\right)+\right.\right.$

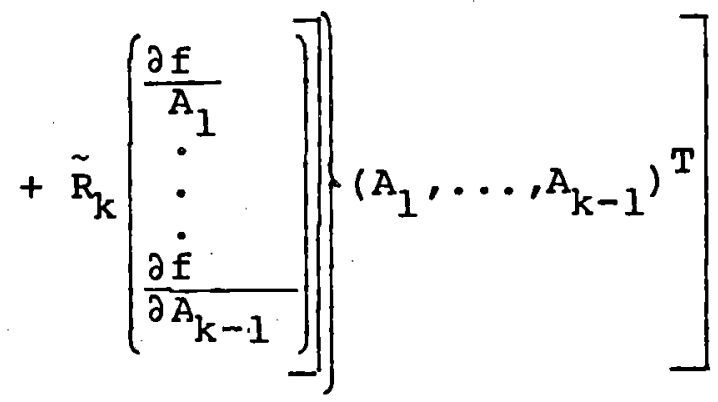

where $M$ is the Hessian associated with (Al1). From the first order conditions (A12) and constant absolute risk aversion, however, this expression is just:

(A15) $\quad \hat{\Sigma}=-E\left\{U^{\prime}\right\} M^{-1}$ 
which is clearly symmetric. Since the choice of which asset to drop via the wealth constraint is arbitrary, this implies that $\sum$ is symmetric also. 
Gramlich, E. and J. Kalchbrenner, 1970. "A Constrained Estimation Approach to the Demand for Liquid Assets." Federal Reserve Board Special Studies Paper No. 3.

Hendershott, P., 1977. Understanding Capital Markets, Vol. 1: A Flow-of-Funds Financial Model. Lexington, Ma.: D. C. Heath \& Co.

Jones, D., 1979. A Structural Econometric Model of the United States Equity Market. Harvard University Ph.D. Thesis.

Merton, R., 1973. "An Intertemporal Capital Asset Pricing Model." Econometrica 4l (September) : 867-87.

Roley, V., 1977. A Structural Model of the U.S. Government Securities Market. Harvard University Ph.D. Thesis.

Silber, W., 1970. Portfolio Behavior and Financial Institutions. New York: Holt, Rhinehart and Winston, Inc.

Smith, G., 1978. "Review of P. Hendershott, Understanding Capital Markets, Vol. 1: A Flow-of-Funds Financial Model." Journal of Monetary Economics 4 (August) : 560-66. 